\title{
Detailed and Direct Observation of Sulfur Crystal Evolution During Operando Analysis of a Li-S Cell with Synchrotron Imaging
}

Sebastian Risse ${ }^{1^{*}}$, Anika Juhl2 ${ }^{2}$, Simone Mascotto ${ }^{2}$, Tobias Arlt ${ }^{3}$, Henning Markötter ${ }^{4}$, André Hilger ${ }^{5}$, Ingo Manke ${ }^{5}$ and Michael Fröba ${ }^{2}$

${ }^{1}$ Helmholtz-Zentrum Berlin, Institute of Soft Matter and Functional Materials, Hahn-Meitner-Platz 1, 14109 Berlin, Germany ${ }^{2}$ Institute of Inorganic and Applied Chemistry, University of Hamburg, Martin-Luther-King Platz 6, 20146 Hamburg, Germany

${ }^{3}$ TU Berlin, Institute of Material Sciences and Technology, Hardenbergstraße 46, 10623 Berlin, Germany

${ }^{4}$ Bundesanstalt für Materialforschung und -prüfung (BAM), Unter den Eichen 87, 12205 Berlin, Germany

${ }^{5}$ Helmholtz-Zentrum Berlin, Institute of Applied Materials, Hahn-Meitner Platz 1, 14109 Berlin, Germany

*Corresponding Author: sebastian.risse@helmholtz-berlin.de

The image post-processing was performed according to the steps in figure S1.

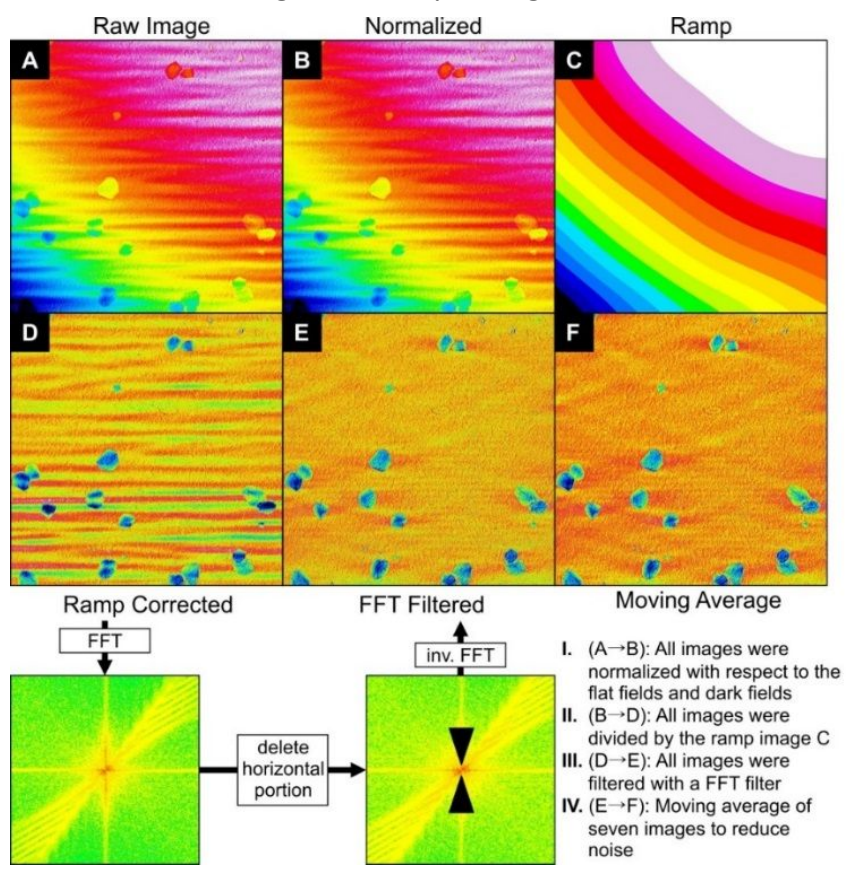

Figure S1 Post-processing procedure for all X-ray radiography images. A: Raw image. B: Darkfield subtracted and flatfield corrected X-ray image. C: Underlying ramp-like attenuation due to inhomogeneous thickness of the monolithic electrode. D: X-ray image divided by the ramp-like attenuation of C. E: Removal of lateral stripes by fast Fourier transform (FFT). F: Moving Average for noise reduction.

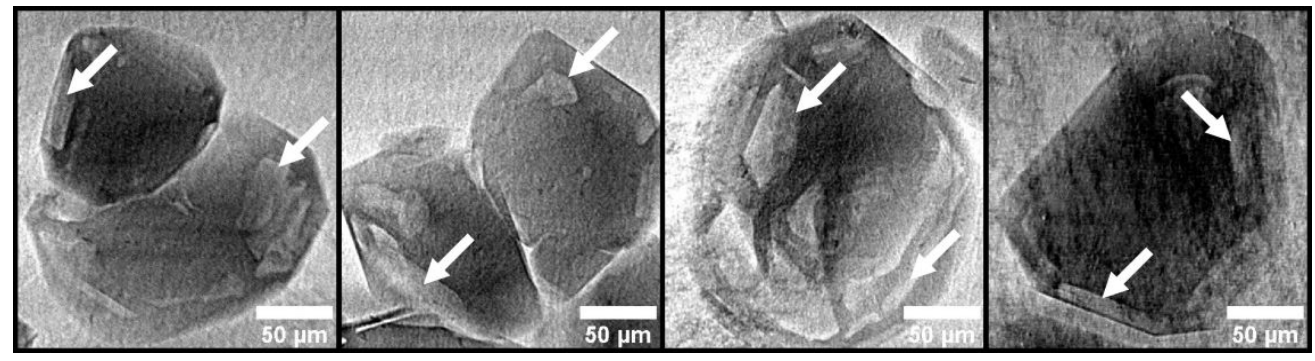

Figure S2 X-ray radiography images of sulfur crystals on the monolithic carbon electrode in the same electrochemical stage like figure $2 \mathrm{~F}(3.0 \mathrm{~V})$ which is the very left one for comparison reasons. The white arrows indicate the areas of crystal imperfections. 


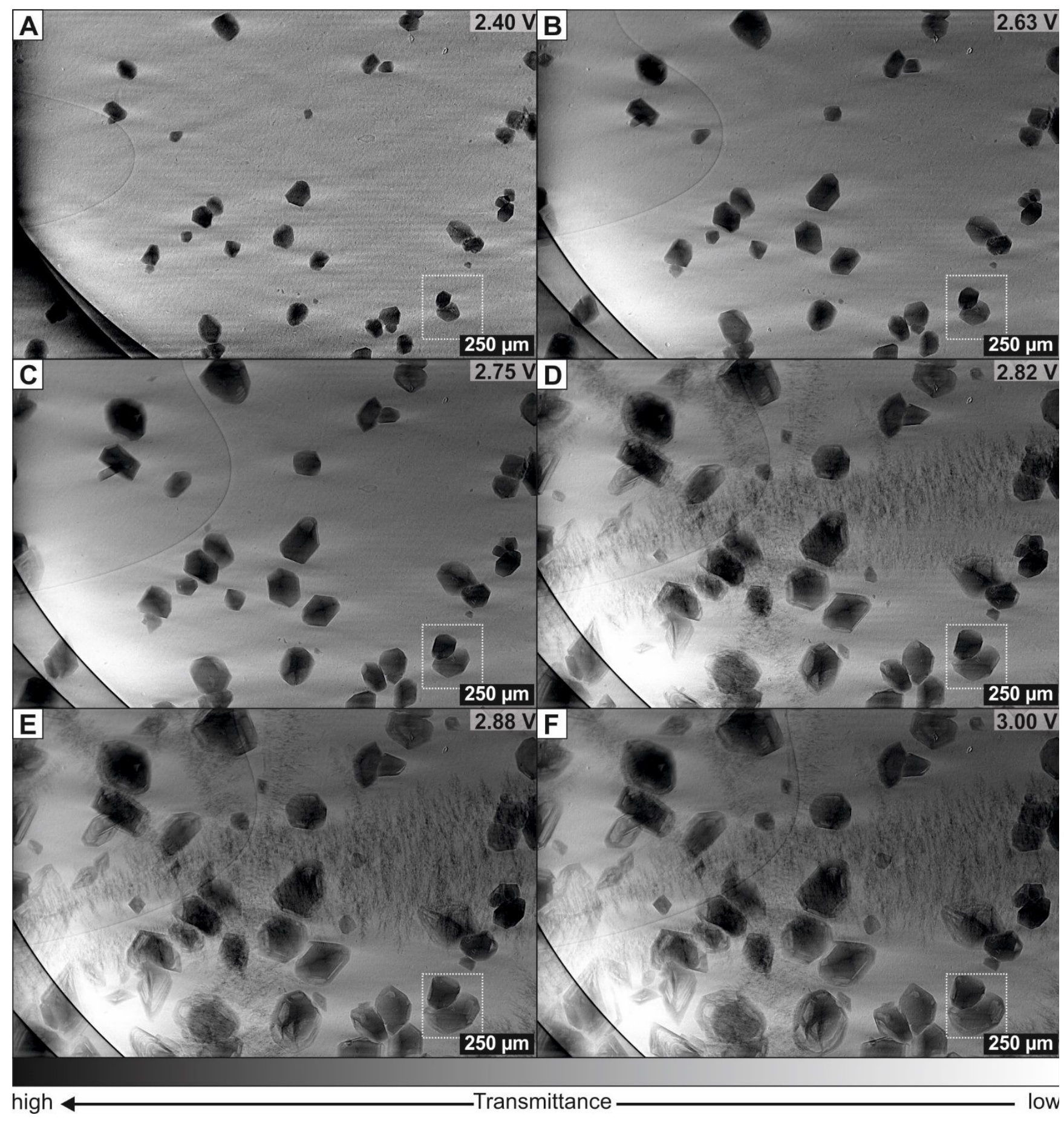

Figure S3 X-ray radiography images of sulfur crystals on the monolithic carbon electrode during the first charge step. The highlighted region (white dashed line) is magnified in figure 2. The images A-F are recorded at the same time as the respective images A-F in figure 2 . 


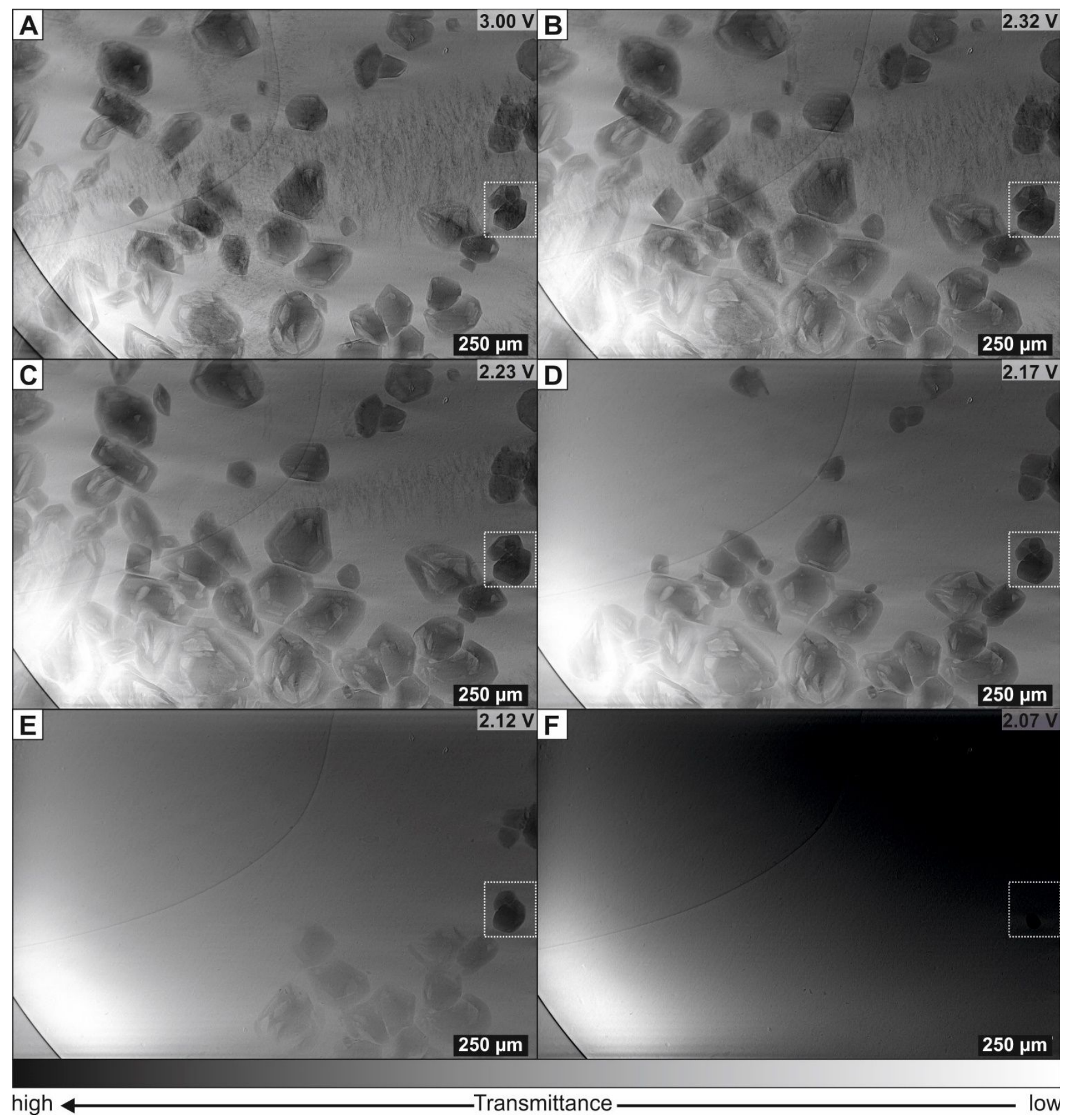

Figure S4 X-ray radiography images of sulfur crystals on the monolithic carbon electrode during the first discharge step. The highlighted region (white dashed line) is magnified in figure 3 . The images A-F are recorded at the same time as the respective images $A-F$ in figure 3. 